\title{
Escleredema Diabeticorum
}

\section{Scleredema Diabeticorum}

\author{
Alejandro Pinzón-Tovar, Harvey Ricardo Cerón-Tapia, Johan Estiven Vargas-Vargas, \\ Mayra Alejandra González-Guzmán, Álvaro Mondragón-Cardona • Neiva (Colombia)
}

DOI: https://doi.org/10.36104/amc.2021.1888

\section{Resumen}

Dentro de los trastornos cutáneos asociados a la diabetes mellitus, se describe el escleredema diabeticorum el cual se caracteriza por un engrosamiento de las capas profundas de la dermis con depósito excesivo de mucina y colágeno, que produce clínicamente endurecimiento de la piel, principalmente en la mitad superior del cuerpo. Se describe un caso clínico correspondiente a un hombre adulto diabético que consultó por la aparición de una lesión indurada en la región cervical con posterior diagnóstico histopatológico de escleroderma diabeticorum. El interés radica en la baja prevalencia de la condición y su asociación al pobre control metabólico de la diabetes. (Acta Med Colomb 2021; 46. DOI: https://doi.org/10.36104/amc.2021.1888).

Palabras clave: escleredema, diabetes mellitus, complicaciones de la diabetes, microangiopatía, mucina.

\begin{abstract}
Scleredema diabeticorum is one of the skin disorders associated with diabetes mellitus, characterized by thickening of the deep layers of the dermis, with excessive mucin and collagen deposition, clinically evidenced in hardening of the skin, especially in the upper half of the body. We describe the clinical case of an adult male diabetic who was seen for an indurated cervical lesion which was subsequently diagnosed histopathologically as scleredema diabeticorum. The interest in this case lies in the low prevalence of the condition and its association with poor metabolic control of diabetes. (Acta Med Colomb 2021; 46. DOI: https://doi.org/10.36104/amc.2021.1888).

Key words: scleredema, diabetes mellitus, diabetes complications, microangiopathy, mucin.
\end{abstract}

Dr. Alejandro Pinzón-Tovar: Especialista en Medicina Interna y Endocrinología, Hospital Universitario Hernando Moncaleano Perdomo, Endho Colombia, Universidad Surcolombiana; Dres. Harvey Ricardo Cerón-Tapia, Johan Estiven Vargas-Vargas y Mayra Alejandra GonzálezGuzmán: Médicos Universidad Surcolombiana; Dr. Álvaro Mondragón-Cardona: Especialista en Medicina Interna, Departamento de Medicina Interna Clínica EMCOSALUD, Hospital Universitario Hernando Moncaleano Perdomo. Universidad Surcolombiana. Neiva (Colombia). Correspondencia: Dr. Álvaro MondragónCardona. Neiva (Colombia).

E-Mail: alvaromondragoncardona@gmail.com Recibido: 21-05-2020 Aceptado: 27-04-2021

\section{Introducción}

El escleredema diabeticorum (ED) es una afección rara del tejido conectivo, con mayor frecuencia afecta pacientes con diabetes mellitus de larga data y con pobre control glucémico; también está asociado a otras entidades como paraproteinemias, enfermedades reumatológicas e infecciones estreptocócicas. Se caracteriza por induración cutánea, simétrica y difusa no depresible, con eritema ocasional y aspecto de piel de naranja que predomina en la zona posterior del cuello, hombros y región superior de la espalda. A continuación, se realiza la presentación de un caso de escleredema asociado a diabetes confirmado por histopatología.

\section{Caso clínico}

Hombre de 68 años con diabetes mellitus tipo 2 diagnosticada hace 37 años y requerimiento de insulina, quien consulta por aparición de lesión pustulosa en región posterior del cuello. Se evidencia foliculitis limitada, siendo lla- mativa la amplia induración de la región cervical posterior y parte superior de la espalda (Figura 1). Al examen físico llamaba la atención la presencia de acantosis nigricans en cuello y axilas, estigmas cutáneos de vasculopatía y neuropatía diabética, datos de disminución de la agudeza visual y fondo de ojo que evidencia retinopatía diabética grado III. A nivel paraclínico su hemoglobina glicosilada era de $13 \%$, con un promedio glucométrico por encima de $270 \mathrm{mg} / \mathrm{dL}$ en las tomas preprandiales y $340 \mathrm{mg} / \mathrm{dL}$ posprandiales. Para su afección cutánea recibió múltiples manejos farmacológicos, incluyendo terapia antimicrobiana sin presentar mejoría, por lo que se decidió toma de biopsia bajo la consideración de un escleredema diabeticorum. El estudio de la patología cutánea demostró aumento del espesor de la dermis, abundantes fibras de colágeno engrosadas y presencia de mucina en las tinciones de azul de toluidina (Figura 2), confirmando así el diagnóstico de escleredema asociado a diabetes mellitus. 


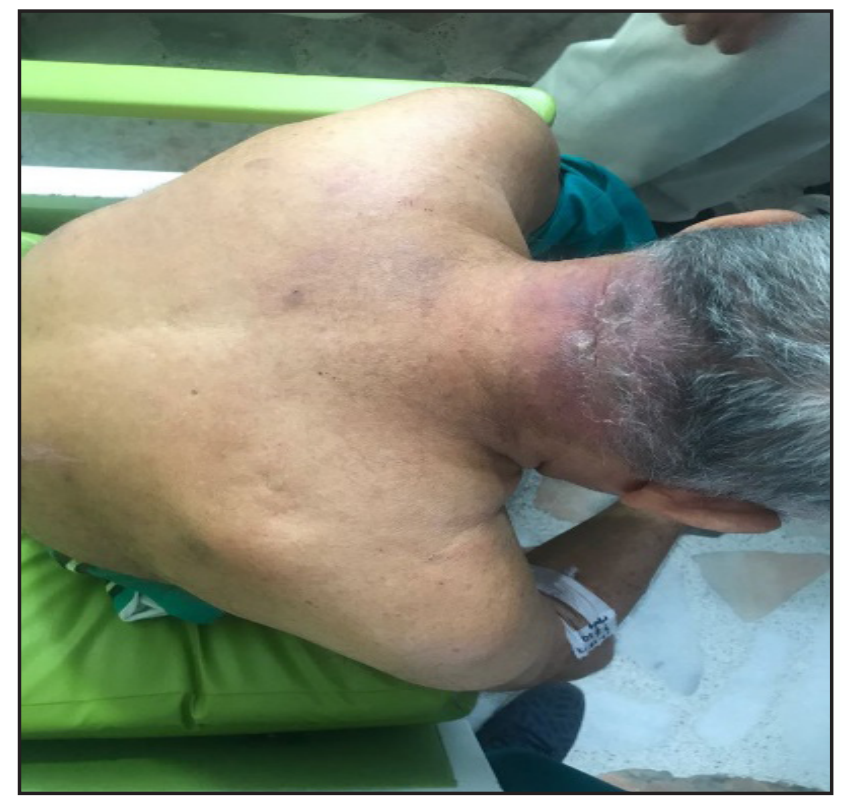

Figura 1. Área de escleredema cervical.

\section{Discusión}

El escleredema es una rara condición de la piel que usualmente se presenta asociada a diabetes mellitus, infecciones o gammapatía monoclonal. Se caracteriza por el desarrollo de una zona de induración difusa, simétrica, que afecta principalmente la parte superior del cuerpo (cabeza, hombros, nuca y miembros superiores $(1,2)$. Su curso clínico es variable y está relacionado de manera directa con las situaciones que lo generan. Puede presentarse de forma aguda o subaguda (dos a seis semanas), en infecciones (usualmente causadas por estreptococos), y generalmente en pacientes con diabetes mellitus mal controlada $(2,3)$.

El escleredema asociado a diabetes mellitus tiene una prevalencia desconocida, con mención importante en reportes o series de casos, restableciéndose que afecta principalmente a individuos adultos, y predominantemente al género masculino $(2,3)$ con obesidad, un sello distintivo de estos pacientes (3). Su causa es desconocida; sin embargo, se encuentra relación con una larga evolución de la enfermedad, obesidad mórbida, y tratamiento con altas dosis de insulina. También se asocia a mal control glucémico y presencia de acantosis nigricans $(3,4)$. Se han podido plantear hipótesis relacionadas con su etiopatogenia con estados hiperglucémicos prolongados que conllevan a la formación irreversible de complejos glicosilados de colágeno y la alteración en la actividad enzimática de las colagenasas dan como resultado acumulación excesiva de colágeno y mucina en la dermis reticular (4). Igualmente se han sugerido alteraciones profibróticas secundarias a hipoxia y daño microvascular (5).

El diagnóstico es clínico, pero se requiere la confirmación mediante el estudio de patología cutánea, en la que se evidencia una dermis aumentada de espesor (hasta tres veces), reducción en el número de glándulas ecrinas, con fibroblastos normales en número y aspecto, fibras de colágeno engrosadas, separadas unas de otras por acúmulos de mucopolisacáridos, especialmente ácido hialurónico y material amorfo que se hacen evidentes con la tinción de azul Alcián y azul de toluidina (6). El curso clínico del escleredema diabeticorum es variable y aunque en la mayoría de los pacientes se presenta de forma persistente, se han observado casos de mejoría clínica (básicamente, disminución de tamaño de la lesión), posterior al adecuado control de la diabetes (7).

En el diagnóstico diferencial de esta patología se deben tener en cuenta principalmente la esclerodermia y escleromixedema, la primera caracterizada por el fenómeno de Raynaud en extremidades y la presencia de telangiectasias, atrofia y calcificaciones (8); y la segunda por ser un tipo de mucinosis que se caracteriza por la presencia de pápulas liquenoides e induración difusa de la piel. También se debe hacer diagnóstico diferencial con otras patologías como son: triquinosis, dermatomiositis, amiloidosis sistémica primaria y la fascitis eosinofílica (9).

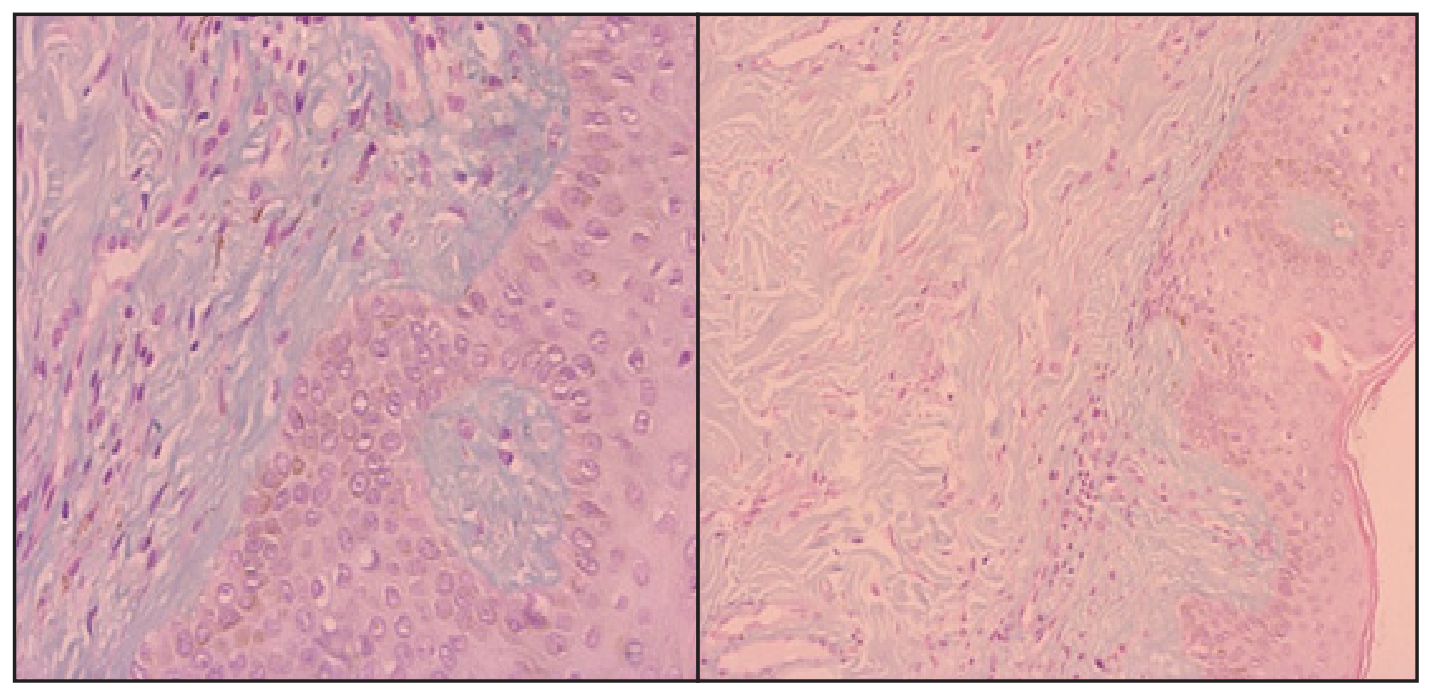

Figura 2. Tinción azul de toluidina con evidencia de depósitos de mucina, engrosamiento de fibras de colágeno y engrosamiento dérmico. 
Aun no se ha establecido un tratamiento específico y efectivo para el escleredema diabeticorum, pero se recomienda la pérdida de peso y un estricto control de la diabetes, que si bien no resuelve el problema sí puede ayudar a detener o mejorar parcialmente la progresión de la lesión cutánea (12). Entre los diferentes tratamientos propuestos, pero con resultados inconstantes encontramos inmunosupresores (como la ciclosporina y metotrexate) en el escleredema asociado a mieloma; fotoquimioterapia con psoraleno $\mathrm{y}$ radiación ultravioleta $\mathrm{A}(\mathrm{PUVA})(10,11)$.

Debe considerarse la manifestación esclerodérmica como marcador de mal control glucométrico y de avance en las complicaciones microvasculares de la enfermedad. Siendo un signo clínico importante a tener en cuenta en la valoración cotidiana de los pacientes con diabetes mellitus en especial con fenómenos de insulinorresistencia.

\section{Referencias}

1. Boin F, Hummers LK. Scleroderma-like Fibrosing Disorders. Rheum Dis Clin North Am. 2008;34(1):199-220. doi:10.1016/j.rdc.2007.11.001

2. Pitarch G, Torrijos A, Martínez-Aparicio A, Vilata J, Fortea J. Escleredema de Buschke asociado a diabetes mellitus. Estudio de cuatro casos. Actas dermo- sifiliogr (Ed impr). 2005;96(1):46-49.

3. Rongioletti F, Kaiser F, Cinotti E, et al. Scleredema. A multicentre study of characteristics, comorbidities, course and therapy in 44 patients. J Eur Acad Dermatology Venereol. 2015;29(12):2399-2404. doi:10.1111/jdv.13272

4. Haustein U. Scleroderma-like lesions in insulin-dependent diabetes mellitus. 1999; 13:50-53.

5. VARGA J, GOTTA S, LI L, SOLLBERG S, LEONARDO M. Scleredema adultorum: case report and demonstration of abnormal expression of extracellular matrix genes in skin fibroblasts in vivo and in vitro. $\mathrm{Br} J$ Dermatol. 2010;132(6):992-999. doi:10.1111/j.1365-2133.1995.tb16962.x

6. Lin IC, Chiu HY, Chan JY, Lin SJ. Extensive scleredema adultorum with loss of eccrine glands. J Am Acad Dermatol. 2014;71(3):e99-e101. doi:10.1016/j. jaad.2014.03.040

7. Rho YW, Suhr KB, Lee JH, Park JK. A clinical observation of scleredema adultorum and its relationship to diabetes. J Dermatol. 1998;25(2):103-107. doi:10.1111/j.1346-8138.1998.tb02358.x

8. Rongioletti F, Rebora A. Updated classification of papular mucinosis, lichen myxedematosus, and scleromyxedema. J Am Acad Dermatol. 2001;44(2):273-281. doi:10.1067/mjd.2001.111630

9. Lewerenz V, Ruzicka T. Scleredema adultorum associated with type 2 diabetes mellitus: a report of three cases. J Eur Acad Dermatol Venereol. 2007;21(4):560 561. doi:10.1111/j.1468-3083.2006.01959.x

10. Doğramaci AC, Inan MU, Atik E, Gökçe C. Scleredema diabeticorum partially treated with low-dose methotrexate: A report of five cases. Balkan Med J. 2012;29(2):218-221. doi:10.5152/balkanmedj.2011.027

11. Kokpol C, Rajatanavin N, Rattanakemakorn P. Successful treatment of scleredema diabeticorum by combining local PUVA and colchicine: A case report. Case Rep Dermatol. 2012;4(3):265-268. doi:10.1159/000345712 